Clinica neurologica dell'Università di Torino (Direttore: Prof. Dino Bolsi)

\title{
Considerazioni sui risultati osservati all'infiltrazione novocainica degli splancnici addominati in un dato tipo di ansiosi
}

\author{
A. G. B. Lusso
}

Si tratta di 20 pazienti raccolti in 10 anni di selezione (di cui il primo trattato del tutto incidentalmente in occasione di una flebite bilaterale della safena curata con il metodo dell'infiltrazione novocaina del simpatico lombare) i quali presentavano crisi di questo tipo: improvviso senso di calore, di costrizione epigastrica con eruttazione; tosto senso di soffocazione con stringimento alla gola, fame d'aria, secchezza alle fauci con necessità di deglutire, ansia vieppiù viva con agitazione, senso di „tensione“; la crisi, della durata di 15 a 25 minuti primi, cessava abitualmente con una scarica alvina ed emissione di abbondanti gas. In ogni caso fu possibile escludere un'affezione del tipo epilettico. In ognuno dei p. dapprima in occasione delle crisi e-poi sistematicamente al mattino vennero eseguite varie infiltrazioni bilaterali della catena simpatica lombare passando con ago da $12 \mathrm{~cm}$ trà le apofisi trasverse della T XII e L I iniettando $20 \mathrm{cc}$ per lato di soluzione di novocaina all' $1 \%$. Con tale tecnica viene infiltrato anche il tessuto lasso nel quale decorrono gli splancnici addominali che così vengono colpiti pure dall'anestetico. Delmas e Laux osservano giustamente che in tale caso è meglio parlare di infiltrazione dèl plesso celiaco più che degli splancnici. In tutti i pazienti venne osservate:

1. l'abortire della crisi all'orchè l'infiltrazione avveniva al suo inizio,

2. l'assenza di crisi per tutta la durata del blocco anestetico ed ancora qualche ore dopo,

3. il perdurare delle crisi in caso di infiltrazione con soluzione indifferente (ad es. soluzione fisiologica) e se l'infiltrazione falliva (test: mancanza di vasodilatazione agli arti inferiori).

Si dovette quindi escludere sia un'azione generale del farmaco di per se, sia uria semplice azione psicoterapia; così il fatto che per tutta la durata dell'anestesia non comparivano crisi stava a dimostrare l'origine primitivamento neuro-vegetativa di esse: viscero-addominale. Il che conferma l'importanza delle alterazioni di funzionalità neuro-vegetativa sul determinismo di elementi sintomatologici delle affezioni del gruppo neurastenico.

Devo dire in appendice che la sospensione del trattamento diede modo alle crisi di ricomparire, che l'esame psichico compiuto anche con il conforto di tests mentali aveva dimostrato una diminuzione netta dei caratteri ansio si ma non la loro scomparsa anche in fase di trattamento.

Anschrift des Verfassers: Prof. A. G. B. Lusso, Clinica neurologica dell'Università di Torino, Torino, Italien. 